\title{
Expressions of Long Non-Coding Rnas in Carcinogenesis of Cervix: A Review
}

\author{
Shrestha Reshies' ${ }^{1}$ Min-Min Yu1,2* \\ ${ }^{1}$ Department of Obstetrics and Gynaecology, the Second Hospital of Nanjing, Affiliated to Medical School of Southeast \\ University, Nanjing, China \\ ${ }^{2}$ Department of Obstetrics and Gynecology, the Second Affiliated Hospital of Nanjing Medical University, Nanjing, China \\ Email: *yuminmin324@126.com
}

How to cite this paper: Reshies, S. and Yu, M.-M. (2018) Expressions of Long NonCoding Rnas in Carcinogenesis of Cervix: A Review. Open Journal of Obstetrics and Gynecology, 8, 130-145.

https://doi.org/10.4236/ojog.2018.82017

Received: January 5, 2018

Accepted: February 6, 2018

Published: February 9, 2018

Copyright $(9) 2018$ by authors and Scientific Research Publishing Inc. This work is licensed under the Creative Commons Attribution International License (CC BY 4.0).

http://creativecommons.org/licenses/by/4.0/

\begin{abstract}
Long non-coding RNAs (lncRNAs) are transcripts longer than 200 nucleotides mostly transcribed by RNA which do not encode proteins. Previously, lncRNAs were considered transcriptional byproducts called "junk DNA" with no biological functions. There are many studies conducted on lncRNAs showing they are actively involved in regulation of epigenetic, transcriptional, and post-transcriptional events. Expressions of lncRNAs are more different in many malignant tumors than in benign tumors and normal tissue. Aberration of lncRNAs is responsible to promote or suppress tumorigenesis and cancer progression. Under different circumstances, lncRNAs exhibit their roles in carcinogenesis such as MALAT1 is responsible for intervening mRNA instability, HOTAIR, MALAT1, ANRIL, PVT1 links with miRNA and histonemodifying complexes, MEG3 associates with miRNA, CCAT2, MEG3, GAS5, UCA1 allies with c-Myc or P53 causing suppression of tumor or oncogenesis. Abnormal expressions of lncRNAs are noticed in gynecological cancers, such as cervical cancer, ovarian cancer, and endometrial cancer. Identification of cervical cancer associated lncRNAs is necessary to understand the molecular biogenesis of cancers. In this review, we summarized the foundation and function of the lncRNAs in terms of tumor progression, invasion, prognosis, apoptosis, metastasis, and chemo-resistance. This review will provide references to determine the clinical applications of lncRNAs as ideal diagnostic biomarkers or therapeutic targets in cervical cancers.
\end{abstract}

\section{Keywords}

lncRNAs, Long Non-Coding RNAs, Cervical Cancer, HPV, HOTAIR, MALAT-1, GAS5, MEG3, PVT1, HULC, ANRIL, CCHE1, CCAT2, UCA1 


\section{Introduction}

Cervical cancer is the second most common cancer and fourth leading cause of mortality in women worldwide [1]. Although the incidence has declined in the developed countries in the past 20 years, it still has a high mortality rate in developing countries. There is high incidence seen in low resource countries, especially in Africa, Latin America, parts of Asia and in some eastern European countries with incidences rising up to $100 / 100,000$. In contrast, the incidence may be as low as 10/100,000 women in developed countries [2]. The lower incidence of cervical cancer in industrialized countries is consequential of awareness and screening programs that were introduced in these countries over several decades ago. Cervical cancer screening then became the prototype of cancer prevention programme by early detection of preneoplastic lesions. Carcinoma in situ is most commonly seen in women aged $35-39$ years likewise the cervical cancer incidence is high in women aged 30 - 34 years $(17 / 1,000,000)$. Almost all invasive cervical carcinomas undergo a stage of the intraepithelial stage, and morphological alterations of early stages of cervical cancer are detectable. According to the epithelial involvement, cervical squamous intraepithelial neoplasia (CIN) is classified into CIN1, CIN2, and CIN3. The transformation from dysplasia to invasive cervical cancer is a very slow progressing process. The development of CIN1 to CIN3 is $10 \%$ and CIN1 to invasive carcinoma is $1 \%$ whereas the rate of progression of CIN2 to CIN3 and cervical cancer are 20\% and 5\% respectively, and CIN3 to invasive cancer is greater than 12\% [3] [4] [5].

To comprehend the molecular pathogenesis of cervical cancer, it is important to understand the HPV infection. Human papillomavirus (HPV) is a doublestranded DNA virus from the papillomavirus family. It is known to be a leading cause of cervical cancer and its precursor lesions. There are 6 early genes (E1, E2, E4, E5, E6, E7) and 2 late genes (L1, L2) in HPV DNA. The early genes regulate the viral replications and the interaction with the host cells. The late genes encrypt for the capsid proteins. Among them, E6 and E7 are known as oncoproteins. Once the virus enters the host cells, E6 and E7 integrate the host DNA and encode for oncoproteins. Subsequently, E6 oncoproteins bind with the host p53 tumor suppressor gene and result in its inactivation. E7 binds to the tumor suppressor gene $\mathrm{pRb}, \mathrm{p} 21, \mathrm{p} 27$ and results in cellular transformation. Till date, more than 200 subtypes of HPV have been identified. It has been categorized into two subtypes: High risk and low-risk types [6] [7]. Persistent infection with high-risk genotypes of the human papillomavirus (hrHPV) has been documented as the principal cause of cervical cancer. $90 \%$ of HPV infection will be eliminated by the host immune system and only minority of women will turn out to develop pre-invasive cancer [8]. Much evidence has shown that human papillomavirus 16 and 18, the hrHPV subtypes are the most common cause of almost $70 \%$ of all cervical cancers [9].

There are about more than 3000 long non-coding RNAs labeled in humans. They are nonprotein coding transcripts mostly transcribed by RNA polymerase 
II, measures longer than 200 nucleotides in size [10] [11]. There are different types of lncRNAs grouped according to their proximity to the closest proteincoding transcripts i.e. sense, antisense, intronic, bidirectional and intergenic. Recent studies have illustrated that LncRNAs are novel nonprotein-coding transcripts, which plays a key role in development and prognosis of cancer but its mechanism requires to be explored in carcinogenesis of different cancer. They act as mRNA sponges and activators to regulate genes expression by operating as competing endogenous RNA(ceRNA) in various biopathophysiological states especially in cancer [12] [13] [14] [15]. There has been many researches and analyses on the involvement of lncRNAs in cervical cancer and evidence reports that majority of lncRNAs may be involved in cervical cancer. IncRNA PVT1, CCAT2, HOTAIR, MALAT-1, ANRIL, UCA1, CCHE1, and HULC have been reported to be upregulated in cervical cancer and promote its progression. In other hand lncRNAs, such as GAS5 and MEG3 are downregulated in cervical cancer and inhibit cervical cancer progression. Based on the biological functions of lncRNAs associated with cervical cancer are summarized in Table 1.

Table 1. Biological functions of lncRNAs associated with cervical cancer.

\begin{tabular}{|c|c|c|c|c|c|c|c|}
\hline Name & Size & Location & Study types and population & Characteristics & Analysis & Functions & References \\
\hline HOTAIR & $2.2 \mathrm{~kb}$ & $12 \mathrm{q} 13.13$ & $\begin{array}{c}\text { Kim, H.J., et al:: Cases: } 111 \\
\text { Control: } 40\end{array}$ & Up-regulation & qRT-PCR & $\begin{array}{c}\text { Cancer progression } \\
\text { Metastasis }\end{array}$ & {$[16][17]$} \\
\hline ANRIL & $3.9 \mathrm{~kb}$ & $9 \mathrm{p} 21.3$ & $\begin{array}{l}\text { Zhang, D., et al:: Cases: } 53 \\
\text { Control: } 53\end{array}$ & Up-regulation & qRT-PCR & Cancer progression & [18] [19] \\
\hline MALAT-1 & $7.5 \mathrm{~kb}$ & $11 \mathrm{q} 13.1$ & $\begin{array}{c}\text { Zhang, Y., et al:: Cases: } 30 \\
\text { Control: } 30\end{array}$ & Up-regulation & qRT-PCR & $\begin{array}{c}\text { Cancer progression } \\
\text { Metastasis }\end{array}$ & {$[20][21]$} \\
\hline UCA1 & $2.3 \mathrm{~kb}$ & 19p13.12 & $\begin{array}{c}\text { Wang, B., et al:: Cases: } 50 \\
\text { Control: } 50\end{array}$ & Up-regulation & qRT-PCR & Cancer progression & [22] [23] \\
\hline PVT1 & $>300 \mathrm{~kb}$ & $8 \mathrm{q} 24$ & $\begin{array}{c}\text { Yang, J., et al:: Cases: } 20 \\
\text { Controls: } 20\end{array}$ & Up-regulation & qRT-PCR & $\begin{array}{c}\text { Cell Proliferation } \\
\text { Metastasis }\end{array}$ & {$[26][27]$} \\
\hline CCAT2 & $0.4 \mathrm{~kb}$ & $8 \mathrm{q} 24.21$ & $\begin{array}{c}\text { Chen, X. et al:: Cases: } 123 \\
\text { Controls: } 123\end{array}$ & Up-regulation & qRT-PCR & $\begin{array}{c}\text { Cancer Progression } \\
\text { Metastasis }\end{array}$ & [28] [29] [30] \\
\hline CCHE1 & $2500 \mathrm{nt}$ & 10 & $\begin{array}{c}\text { Yang, M., et al:: Cases: } 141 \\
\text { Controls: } 141\end{array}$ & Up-regulation & qRT-PCR & Cell Proliferation & {$[31][32]$} \\
\hline HULC & $0.5 \mathrm{~kb}$ & $6 \mathrm{p} 24.3$ & $\begin{array}{c}\text { Wang, Y., et al: Cases: } 244 \\
\text { Controls: } 244\end{array}$ & Up-regulation & qRT-PCR & Cancer Progression & {$[33][34]$} \\
\hline GAS5 & $\begin{array}{l}\text { Multiple } \\
\text { lncRNA and } \\
\text { snoRNAs }\end{array}$ & $1 q 25.1$ & $\begin{array}{c}\text { Cao, S., et al.: Cases: } 102 \\
\text { Controls: } 102\end{array}$ & Down-regulation & qRT-PCR & Tumor Suppressor & {$[35][36]$} \\
\hline
\end{tabular}




\section{Roles of IncRNAs in Cervical Cancer}

\subsection{MALAT-1}

Metastasis-associated lung adenocarcinoma transcript 1(MALAT1) is a large, infrequently spliced $7.5 \mathrm{~kb}$ in length lncRNA located in chr11q13. MALAT1 was first identified by its link in non-small cell lung cancer (NSCLC) in 2003 [20]. A larger number of studies have illustrated that lncRNA MALAT1 is upregulated in many types of tumor and contributes to tumor cell proliferation, invasion apoptosis, and migration. A recent study found that HPV infection known as a leading cause of development of cervical cancer is associated with significantly increased MALAT1 expression [20] [37]. Evidence illustrate high levels of MALAT1 are aberrantly expressed in cancer tissues compared to normal cervical tissues and is associated with a poor prognosis. MALAT1 is overly expressed in cervical cancer and consequently stimulates tumor growth and invasion and also inhibiting apoptosis. Downregulation of MALAT-1 induces the expression of caspase-3, caspase- 8 and Bcl-2. Evidence demonstrates the depletion of MALAT-1 activated expression of p53 ensuing cell cycle arrest and apoptosis. Knockdown of MALAT1 simultaneously reduces the expressions of cell cycle regulatory molecules cyclin D1, cyclin E and CDK6 leading to enhanced cells arrested in G1 phase [21] [37] [38].

\subsection{HOTAIR}

HOX transcript antisense intergenic RNA (HOTAIR) is $2.2 \mathrm{~kb}$ long and has 6 exons. It is located at the antisense strand of the HOXC gene cluster in chromosome 12q13.13. It was first identified from HOXC gene cluster for its involvement in the determining the proximal-distal axis during development [39] [40] [41]. HOTAIR interacts with PRC2 to target the HOXD locus, resulting in $\mathrm{H} 3 \mathrm{~K} 27$-trimethylation and gene silencing by histone methyltransferase EZH2, signifies that HOTAIR selectively targets PRC2 complex to silence the transcription of HOXD locus [39]. VEGF and MMP-9 play a crucial role in developing a tumor by increasing cell migration and invasion. In cervical cancer expression of HOTAIR is associated with VEGF and MMP-9 explaining the high expression of HOTAIR upregulates VEGF and MMP-9 resulting in progression of cervical cancer. HOTAIR also has a role play in recurrence of cervical cancer. Low expression of HOTAIR inhibits cellular proliferation, migration, and invasion of cervical cancer. EmT is also important in cell migration and invasion. EMT-related genes in cervical cancer were reversed with the inhibition of HOTAIR [17] [42].

\subsection{ANRIL}

Antisense Non-coding RNA in the INK4 Locus (ANRIL) is a newly discovered lncRNA. It is $3.8 \mathrm{~kb}$ in length. It is located in the $9 \mathrm{p} 21$ chromosomal region. It is transcribed in the opposite direction from its neighboring INK4B/ARF-INK4A 
gene cluster. ANRIL was first identified in a genetic analysis of melanoma with neural tumors [18]. ANRIL is intensely associated with a genetic marker of coronary artery disease and also upregulated in prostate cancer [18]. The loci where ANRIL is located is highly susceptible to cardiovascular disease [43] [44], few cancers [45] [46] [47] [48] and other clinical conditions [43] [49] [50]. ANRIL epigenetically inhibits tumor suppressors CDKN2A (p16) and CDKN2B (p15), and in doing so it plays a substantial role in cellular proliferation and senescence [51] [52] [53]. Tumor suppressors CDKN2A (p16), ARF (p14) and CDKN2B (p15) have a major participation in cell proliferation, apoptosis, and senescence. ANRIL is involved in suppression of p16 (INK4A), p14 (ARF), p15 (INK4B). ANRIL is interrelated with the advancement of cell cycle progression and suppression of apoptosis and senescence. Depletion of tumor suppressor INK4B (p15) and upregulation of the anti-apoptotic Bcl-2 explains ANRIL enhances cell proliferation [54]. Low expression of ANRIL upregulates tumor suppressors p15 levels but shows no influence in the expression of p16 or p14 causing cell-cycle arrest at the G2/M phase, facilitating growth suppression [55]. A recent study exhibits that expression of pro-oncogenic protein Ras stimulates the expression of p15INK4B and p16INK4A ensuing inhibition on ANRIL expression [53]. ANRIL overexpression is directly associated with the advanced FIGO stage, lymph node metastasis and poor overall survival in cervical cancer. Signaling pathway PI3K/Akt is an important process in proliferation and EMT in carcinogenesis. Downregulation of ANRIL includes a significant decrease in the expression of phosphorylated(p)-PI3K and p-Akt, suggesting a contribution of PI3K/Akt pathway in the progression of ANRIL-induced cervical cancer. Likewise knockdown of ANRIL leads to cervical cancer cell proliferation and metastasis by inactivation of the PI3K/Akt pathway [19].

\subsection{MEG3}

Maternally expressed gene (MEG3) lncRNA is $1.6 \mathrm{~kb}$ in length consisting of 10 exons, belonging to DLK1-MEG3 locus imprinted in human chromosome 14q32.3. It was first identified as the human homolog of Gtl2 gene (gene trap locus 2) encrypted on chromosome 12 of a mouse [24] [56]. Many normal tissues express lncRNA $M E G 3$, especially, the brain and pituitary gland [57]. The various mechanisms responsible for lack of MEG3 expressions in cancers are hypermethylation in the MEG3 regulatory regions and IG-DMR, gene eliminations and also miRNA induced post-transcriptional degradation [58] [59]. A transcription factor, TP53 is closely associated with the development and progression of the tumor. Overexpression of MEG3 results in a significant rise in expression of a potent tumor suppressor p53 (TP53), playing a central role in arresting the cell cycle and apoptosis signaling [60]. Increased expressions of MEG33 results in activation of p53 inhibiting cellular proliferation and inducing apoptosis in cancer cells suggesting its role as a tumor suppressor [61]. Retinoblastoma (RB1) is another important tumor suppressor involved in cell cycle progression, cell dif- 
ferentiation, and apoptosis. Activation of tumor suppressor pRB diminishes expression of DNMT1 resulting in an increase of MEG3 expression which ultimately decreases cell growth [62]. Downregulation of MEG3 strongly correlates with advanced with advanced FIGO stage, enlarged tumor size, depth of tumor, metastasis to lymph nodes and presence of hrHPV [25]. Cervical cancer tissues markedly expressed lower levels of MEG3 compared to the adjacent normal tissue. Likewise over expression of MEG3 exhibit strikingly suppression in growth and increased apoptosis by $\mathrm{p} 53$ and caspase which convinced it as a tumor suppressor [63] [64]. Therefore these findings determine MEG3 is one of the lncRNAs with tumor suppressor activity.

\subsection{GAS5}

Growth arrest-specific transcript 5 (GAS5) lncRNA has many small nucleolar RNAs (snoRNAs), microRNAs (miRNAs) and PIWI-interacting RNAs (piRNAs) located in the 1q25 chromosomal region which was initially extracted from mouse NIH 3T3 cells using subtraction hybridization [35] [65]. There are many pieces of evidence showing that downregulation of GAS5 acts as a tumor suppressor in various types of cancers in humans, such as breast cancer, prostate cancer, and lung cancer [66] [67] [68]. Cyclin-dependent kinase 6 (CDK6) is a GAS-5 associated protein which enhances cell cycle progression in bladder cancer. The decrease in the expression of GAS- 5 considerably increased CDK6 expressions reducing G0/G1 phase significantly and escalating $S$ phase confirms GAS-5 regulates CDK6 [69]. Due to its tumor suppressive character GAS5 is considered as a rising star among all the lncRNAs. When GAS5 expression was decreased interestingly there was an increase in the expression of miR-21. GAS5 induced apoptosis and significantly suppressed cell growth, tumor invasion, and lymph node metastasis in cervical cancer [36]. Signaling pathway P13K/Akt is an important regulator for promoting cellular proliferation, cellular growth and survival. Some study reports P13K/Akt has a significant part in cisplatin-resistance cancer cells [70]. Downregulation of GAS5 increased expression of miR-21 causing decreases in PTEN, it's one of the genes in the PI3K/Akt pathway activating the PI3K/Akt pathway. Therefore, low expression of GAS5 increases the expression of miR-21which is responsible for regulation of PTEN explaining the cisplatin resistance in cervical cancer cells [71]. Therefore this evidence confirms GAS5 is an independent marker for predicting the clinical outcome in cervical cancer.

\subsection{CCAT2}

Colon cancer-associated transcript 2 (CCAT2) is $0.4 \mathrm{~kb}$ long lncRNA located in the 8q24.21 chromosomal region. It was first discovered in colorectal cancer in 2013 [28]. Many studies are evident that CCAT2 promotes chromosomal instability, cellular progression and tumor metastasis in various kinds of cancer such as colon [28], lung cancer [72] breast cancer [73], gastric cancer [74], ovary can- 
cer [75] and cervical cancer [30]. Knockdown of CCAT2 inhibited the cell development, growth and promoted cell death [29]. CCAT2 upregulates MYCregulated miRNA-17-5p and miRNA-20a responsible for cellular proliferation, chromosomal modifications, and metastasis in many cancers via [76]. Dysregulation activation of the $\mathrm{Wnt} / \beta$-catenin signaling pathway contributes the development of human cancers [77]. Transcription factor 7-like 2(TCF7L2) is known to be an inducer of $\mathrm{Wnt} / \beta$-catenin signaling pathway. CCAT2 binds with TCF7L2, upregulates MYC, miR-17-5p, and miR20a expressions triggering Wnt signaling pathway [78]. The upregulation of CCAT2 positively influences the $\mathrm{Wnt} / \beta$-catenin signaling pathway to promote proliferation and metastasis of cancer cells [79]. The expression of CCAT2 was relatively high in cervical cancer tissues than normal tissues. [30]. CCAT2 expression was highly dependent on cervical invasion depth, FIGO stage, and lymph node metastasis. The correlation between CCAT2 and metastasis reveals a poor prognosis in cervical cancer [80]. Therefore, overexpression of CCAT2 was theorized as an independent prognostic factor for poor overall survival in cervical cancer. Additionally, knockdown of CCAT2 by transfection of siRNA induced $G_{0} / G_{1}$ phase cell cycle arrest and apoptosis [81].

\subsection{UCA1}

Urothelial cancer associated 1(UCA1) is $2.3 \mathrm{~kb}$ long located in 19p13.12 encodes 3 isoforms (1.4, 2.2 and $2.7 \mathrm{~kb}$ ) and expresses 2 transcripts. The isoform $2.2 \mathrm{~kb}$ in length has been identified as drug-resistant (CUDR) [22] [82] Since CUDR is not so easily detected in normal tissues and due to its relatively low expression than other biomarkers of cancer, it can be an effective biomarker to identify the development of cancer and its therapeutic responses [83]. In 2006 UCA1 was initially identified in human bladder transitional cell carcinoma with high specificity and sensitivity [82] [84]. Growing evidences have reported that UCA1 is abundant in various kinds of cancers, including bladder cancer, colorectal cancer, esophageal squamous cell carcinoma, tongue squamous cell carcinoma, breast cancer, gastric cancer, ovarian cancer, cervical cancer and melanoma [23] [84][90]. Aberrant expressions of UCA1 in cervical cancer suggests that it encourages cisplatin resistance in cervical cancer cells through signaling pathways regulating cell apoptosis, which are involved in balancing expression of caspase-3, p21, CDK2, and survivin. Increased level of survivin and decreased the level of p21 boosted cellular proliferation and also downregulating caspase 3 and up-regulating CDK2 suppressed apoptosis representing UCA1 has crucial regulatory mechanisms in the cisplatin-resistance in cervical cancer cells [91]. Thus the provided evidence supports that UCA1 can be used as an effective biomarker for therapeutic strategy and to identify the development of cervical cancer.

\subsection{PVT1}

Plasmacytoma variant translocation 1 (PVT1) is a $>300 \mathrm{~kb}$ long intergenic 
lncRNA located in the 8q24 chromosomal region of the human genome which is also the commonest sites of cancer-related amplifications containing both $M Y C$ and PVT1 [92] [93]. PVT1 involvement in carcinogenesis was first demonstrated by frequent translocations in mouse plasmacytomas and Burkitt's lymphomas in human [94] [95] [96]. Many reports have shown significant overexpression of PVT1 in relation with risk, recurrence, and survival in many cancers [26] [93] [97]-[102]. PVT1 has gained attraction from the cancer field due to its frequent co-amplification with $M Y C$ in several solid tumors. Assembled evidence reported that more than $45 \%$ of 500 ovarian cancers have co-amplification with PVT1 and MYC. According to the TCGA report from cBioPortal 13\% of 40 cervical cancer tumors have highly expressed P VT1, however, co-amplification of PVT1 and MYC only occurs in 27.5\% (11/40 cases) [103] [104]. Overexpressed P VT1 in cervical cancer tissue correlated to FIGO stage, tumor size, and poor prognosis. Up-regulation of PVT1 with a significantly higher expression of miRNAs 1204 and 1206 in cervical cancer cells demonstrated cellular proliferation, cell cycle progression, and migration. Through changing histone methylation PVT1 inhibits miR-200b expression. PVT1 associates with EZH2 epigenetically silencing miR-200b expression in cervical cancer ensuing cellular proliferation, cell cycle progression, and migration of cervical cancer cells [105]. As a result, knockdown of PVT1 in cervical cancer cells decreased proliferation, migration and invasion and increased apoptosis and cisplatin cytotoxicity, suggesting that PVT1 functions as a multidimensional role in cervical carcinogenesis [106]. In a cohort study, the PVT1 level was measured in cervical cancer, cervical intraepithelial neoplasia (CIN) and normal cells; the result showed that serum PVT1 is high in cervical cancer and distinctly distinguishes cervical cancer suggesting PVT1 may be a novel noninvasive diagnostic biomarker for cervical cancer [27].

\subsection{CCHE1}

Cervical carcinoma high-expressed 1 (CCHE1) lncRNA is 2500 nucleotides long and is located on chromosome 10. Upregulation of CCHE1 is significantly correlated with advanced FIGO stages, increased tumor size, invasion, and prognosis in cervical cancer. CCHE1 binds to physically associates with proliferating cell nuclear antigen (PCNA)mRNA, promoting its expressions and consequently increasing proliferation of cervical cancer cells. In contrast, depletion of PCNA stamp out the effects of CCHE1 on the proliferation of cervical cancer cells thus indicating that CCHE1 could be a prognostic factor and therapeutic target for cervical cancer [31].

\subsection{HULC}

Highly up-regulated in liver cancer (HULC) is a recently found lncRNA $0.5 \mathrm{~kb}$ in length encoded in chromosome 6p24.3. HULC was found originally in hepatocellular carcinoma and is also highly expressed in various tumors, such as he- 
patocellular carcinoma, colorectal carcinoma, osteosarcoma, gastric cancer, and large B-cell lymphoma. Abnormal expression of HULC was associated with metastasis and prognosis of cancers hence, it established its function in various carcinogenesis [33] [107] [108] [109] [110]. HULC expressions with FIGO stage, lymph nodes metastasis, the cervical invasion was significantly demonstrated in association with univariate analysis. Multivariate analysis indicated that expression of HULC appeared as an independent factor associated with five-year survival rates. The overall survival rates of cervical cancer patient were closely correlated to overexpression of HULC [34].

\section{Conclusion}

Aberrant expressions of lncRNAs have been noted in many types of cervical cancer. Identification of cervical cancer associated lncRNAs is important for understanding the molecular biogenesis of cervical cancer. Studies on the association of lncRNAs in cervical cancer are still in its preliminary stages. Accumulating evidence postulates that dysregulated expressions of lncRNAs have been linked to clinicopathological features in cervical cancer. However, not so much is known about the impact of lncRNAs expressions on cell proliferation, metastasis, and apoptosis in cervical cancer. Evidence regarding the long non-coding RNA expression in cervical cancer reveals that the configuration of lncRNA expression in the cervical cancer cell and precancerous lesions provides important information about its role in cancer initiation, tumor progression, and metastatic spread. There is a large scale of studies investigating the long non-coding RNA expressions in different kinds of cancer. Expression pattern of many lncRNAs needs to be mapped and be further studied to understand its functions and expressions in cervical cancer. The lncRNAs mentioned in this review have several strong evidences providing the needed information to justify its expressions in cervical cancer. The detailed understanding of its mechanism and functions may lead to the identification of new liabilities in cervical cancer. The identification of lncRNA involved in carcinogenesis, tumor suppression, or metastasis provides new prospects to develop novel therapeutics of cancer by targeting those lncRNAs. Drug-resistance is a major challenge that confines the efficacy of treatment in cancer. IncRNAs conferring the drug-resistance phenotype are appreciated for its outcome. DNA repair and cell cycle progression, sensitivity to apoptotic-effect, the involvement of lncRNA in modulating signaling pathways, drug transporter expression and elimination are recognized as resistance therapeutic measures. A massive number of studies have been seeking for novel diagnostic biomarkers for cervical cancer; lncRNAs have shown its potentials as independent biomarkers for early diagnosis and prognostic factor. In the future lncRNAs might serve as early diagnostic biomarkers and novel targets for early prophylaxis and effective treatment and gene therapy of cervical cancer. The use of lncRNAs as biomarkers has prompted considerable interest in researchers and it can be expected that in the upcoming years, some lncRNAs might become useful biomarkers for prognosis and effective treatment to cervical cancer. 


\section{References}

[1] Jemal, A., et al. (2011) Global Cancer Statistics. CA: A Cancer Journal for Clinicians, 61, 69-90. https://doi.org/10.3322/caac.20107

[2] Shanta, V., et al. (2000) Epidemiology of Cancer of the Cervix: Global and National Perspective. Journal of the Indian Medical Association, 98, 49-52.

[3] Bosch, F.X., et al. (2002) The Causal Relation between Human Papillomavirus and Cervical Cancer. Journal of Clinical Pathology, 55, 244-265.

[4] Munoz, N., et al. (1992) The Causal Link between Human Papillomavirus and Invasive Cervical Cancer: A Population-Based Case-Control Study in Colombia and Spain. International Journal of Cancer, 52, 743-749.

https://doi.org/10.1002/ijc.2910520513

[5] Schiffman, M.H., et al. (1993) Epidemiologic Evidence Showing that Human Papillomavirus Infection Causes Most Cervical Intraepithelial Neoplasia. JNCI: Journal of the National Cancer Institute, 85, 958-964. https://doi.org/10.1093/jnci/85.12.958

[6] Dueñas-González, A., et al. (2005) Epigenetics of Cervical Cancer. An Overview and Therapeutic Perspectives. Molecular Cancer, 4, 38.

https://doi.org/10.1186/1476-4598-4-38

[7] Smith, J.S., et al. (2007) Human Papillomavirus Type Distribution in Invasive Cervical Cancer and High-Grade Cervical Lesions: A Meta-Analysis Update. International Journal of Cancer, 121, 621-632. https://doi.org/10.1002/ijc.22527

[8] Pagliusi, S.R. and Aguado, M.T. (2004) Efficacy and Other Milestones for Human Papillomavirus Vaccine Introduction. Vaccine, 23, 569-578.

https://doi.org/10.1016/j.vaccine.2004.07.046

[9] Franco, E.L., et al. (1999) Epidemiology of Acquisition and Clearance of Cervical Human Papillomavirus Infection in Women from a High-Risk Area for Cervical Cancer. The Journal of Infectious Diseases, 180, 1415-1423.

https://doi.org/10.1086/315086

[10] Derrien, T., et al. (2012) The GENCODE v7 Catalog of Human Long Noncoding RNAs: Analysis of Their Gene Structure, Evolution, and Expression. Genome Research, 22, 1775-1789. https://doi.org/10.1101/gr.132159.111

[11] Mercer, T.R., Dinger, M.E. and Mattick, J.S. (2009) Long Non-Coding RNAs: Insights into Functions. Nature Reviews Genetics, 10, 155-159.

https://doi.org/10.1038/nrg2521

[12] Wang, K.C. and Chang, H.Y. (2011) Molecular Mechanisms of Long Noncoding RNAs. Molecular Cell, 43, 904-914. https://doi.org/10.1016/j.molcel.2011.08.018

[13] Mercer, T.R. and Mattick, J.S. (2013) Structure and Function of Long Noncoding RNAs in Epigenetic Regulation. Nature Structural \& Molecular Biology, 20, 300307. https://doi.org/10.1038/nsmb.2480

[14] Li, J.-H., et al. (2013) starBase v2.0: Decoding miRNA-ceRNA, miRNA-ncRNA and Protein-RNA Interaction Networks from Large-Scale CLIP-Seq Data. Nucleic Acids Research, 42, D92-D97. https://doi.org/10.1093/nar/gkt1248

[15] Klattenhoff, C.A., et al. (2013) Braveheart, a Long Noncoding RNA Required for Cardiovascular Lineage Commitment. Cell, 152, 570-583. https://doi.org/10.1016/j.cell.2013.01.003

[16] Bhan, A. and Mandal, S.S. (2015) lncRNA HOTAIR: A Master Regulator of Chromatin Dynamics and Cancer. Biochimica et Biophysica Acta (BBA)-Reviews on Cancer, 1856, 151-164. https://doi.org/10.1016/j.bbcan.2015.07.001

[17] KIm, H.J., et al. (2015) Long Non-Coding RNA HOTAIR Is Associated with Hu- 
man Cervical Cancer Progression. International Journal of Oncology, 46, 521-530. https://doi.org/10.3892/ijo.2014.2758

[18] Pasmant, E., et al. (2007) Characterization of a Germ-Line Deletion, Including the Entire INK4/ARF Locus, in a Melanoma-Neural System Tumor Family: Identification of ANRIL, an Antisense Noncoding RNA Whose Expression Coclusters with ARF. Cancer Research, 67, 3963-3969. https://doi.org/10.1158/0008-5472.CAN-06-2004

[19] Zhang, D., et al. (2017) Long Non-Coding RNA ANRIL Indicates a Poor Prognosis of Cervical Cancer and Promotes Carcinogenesis via PI3K/Akt Pathways. Biomedicine \& Pharmacotherapy, 85, 511-516. https://doi.org/10.1016/j.biopha.2016.11.058

[20] Ji, P., et al. (2003) MALAT-1, a Novel Noncoding RNA, and Thymosin $\beta 4$ Predict Metastasis and Survival in Early-Stage Non-Small Cell Lung Cancer. Oncogene, 22, 8031-8041. https://doi.org/10.1038/sj.onc.1206928

[21] Zhang, Y., et al. (2015) Human MALAT-1 Long Non-Coding RNA Is Overexpressed in Cervical Cancer Metastasis and Promotes Cell Proliferation, Invasion and Migration. J. Buon, 20, 1497-1503.

[22] Wang, Y., et al. (2012) Long Non-Coding RNA UCA1a (CUDR) Promotes Proliferation and Tumorigenesis of Bladder Cancer. International Journal of Oncology, 41, 276-284.

[23] Han, Y., et al. (2014) UCA1, a Long Non-Coding RNA Up-Regulated in Colorectal Cancer Influences Cell Proliferation, Apoptosis and Cell Cycle Distribution. Pathology-Journal of the RCPA, 46, 396-401.

[24] Miyoshi, N., et al. (2000) Identification of an Imprinted Gene, Meg3/Gtl2 and Its Human Homologue MEG3, First Mapped on Mouse Distal Chromosome 12 and Human Chromosome 14q. Genes to Cells, 5, 211-220. https://doi.org/10.1046/j.1365-2443.2000.00320.x

[25] Zhang, J., et al. (2017) Downregulation of Long Noncoding RNA MEG3 Is Associated with Poor Prognosis and Promoter Hypermethylation in Cervical Cancer. Journal of Experimental \& Clinical Cancer Research, 36, 5. https://doi.org/10.1186/s13046-016-0472-2

[26] Yang, Y.-R., et al. (2014) Increased Expression of The lncRNA PVT1 Promotes Tumorigenesis in Non-Small Cell Lung Cancer. International Journal of Clinical and Experimental Pathology, 7, 6929.

[27] Yang, J., et al. (2016) Long Noncoding RNA PVT1 as a Novel Serum Biomarker for Detection of Cervical Cancer. European Review for Medical and Pharmacological Sciences, 20, 3980-3986.

[28] Ling, H., et al. (2013) CCAT2, a Novel Noncoding RNA Mapping to 8q24, Underlies Metastatic Progression and Chromosomal Instability in Colon Cancer. Genome Research, 23, 1446-1461. https://doi.org/10.1101/gr.152942.112

[29] Wang, D., et al. (2017) Long Noncoding RNA CCAT2 as a Novel Biomaker of Metastasis and Prognosis in Human Cancer: A Meta-Analysis. Oncotarget, 8, 75664. https://doi.org/10.18632/oncotarget.18161

[30] Chen, X., Liu, L. and Zhu, W. (2015) Up-Regulation of Long Non-Coding RNA CCAT2 Correlates with Tumor Metastasis and Poor Prognosis in Cervical Squamous Cell Cancer Patients. International Journal of Clinical and Experimental Pathology, 8, 13261.

[31] Yang, M., et al. (2015) Long Noncoding RNA CCHE1 Promotes Cervical Cancer Cell Proliferation via Upregulating PCNA. Tumor Biology, 36, 7615-7622. https://doi.org/10.1007/s13277-015-3465-4 
[32] Chen, Y., et al. (2017) Long Non-Coding RNA CCHE1 Overexpression Predicts a Poor Prognosis for Cervical Cancer. European Review for Medical and Pharmacological Sciences, 21, 479-483.

[33] Peng, W., Wu, J. and Feng, J. (2016) Long Noncoding RNA HULC Predicts Poor Clinical Outcome and Represents Pro-Oncogenic Activity in Diffuse Large B-Cell Lymphoma. Biomedicine \& Pharmacotherapy, 79, 188-193. https://doi.org/10.1016/j.biopha.2016.02.032

[34] Wang, Y., et al. (2016) Expression of lncRNA HULC in Cervical Cancer and Its Correlation with Tumor Progression and Patient Survival. European Review for Medical and Pharmacological Sciences, 20, 3987-3991.

[35] Li, W., et al. (2017) Systematic Review and Meta-Analysis of the Utility of Long Non-Coding RNA GAS5 as a Diagnostic and Prognostic Cancer Biomarker. Oncotarget, 8, 66414. https://doi.org/10.18632/oncotarget.19040

[36] Cao, S., et al. (2014) Decreased Expression of lncRNA GAS5 Predicts a Poor Prognosis in Cervical Cancer. International Journal of Clinical and Experimental Patho$\log y, 7,6776$.

[37] Guo, F., et al. (2010) Inhibition of Metastasis-Associated Lung Adenocarcinoma Transcript 1 in Caski Human Cervical Cancer Cells Suppresses Cell Proliferation and Invasion. Acta Biochimica et Biophysica Sinica, 42, 224-229.

https://doi.org/10.1093/abbs/gmq008

[38] Yang, L., et al. (2015) High MALAT1 Expression Predicts a Poor Prognosis of Cervical Cancer and Promotes Cancer Cell Growth and Invasion. European Review for Medical and Pharmacological Sciences, 19, 3187-3193.

[39] Rinn, J.L., et al. (2007) Functional Demarcation of Active and Silent Chromatin Domains in Human HOX Loci by Noncoding RNAs. Cell, 129, 1311-1323. https://doi.org/10.1016/j.cell.2007.05.022

[40] Hung, T. and Chang, H.Y. (2010) Long Noncoding RNA in Genome Regulation: Prospects and Mechanisms. RNA Biology, 7, 582-585. https://doi.org/10.4161/rna.7.5.13216

[41] Nie, Y., et al. (2013) Long Non-Coding RNA HOTAIR Is an Independent Prognostic Marker for Nasopharyngeal Carcinoma Progression and Survival. Cancer Science, 104, 458-464. https://doi.org/10.1111/cas.12092

[42] Kim, H., et al. (2014) Long Noncoding RNA HOTAIR Is Associated with Human Cervical Cancer Progression. Gynecologic Oncology, 133, 5. https://doi.org/10.1016/j.ygyno.2014.03.027

[43] Burton, P.R., et al. (2007) Genome-Wide Association Study of 14,000 Cases of Seven Common Diseases and 3,000 Shared Controls. Nature, 447, 661-678. https://doi.org/10.1038/nature05911

[44] Broadbent, H.M., et al. (2007) Susceptibility to Coronary Artery Disease and Diabetes Is Encoded by Distinct, Tightly Linked SNPs in the ANRIL Locus on Chromosome 9p. Human Molecular Genetics, 17, 806-814. https://doi.org/10.1093/hmg/ddm352

[45] Sherborne, A.L., et al. (2010) Variation in CDKN2A at 9p21.3 Influences Childhood Acute Lymphoblastic Leukemia Risk. Nature Genetics, 42, 492-494. https://doi.org/10.1038/ng.585

[46] Turnbull, C., et al. (2010) Genome-Wide Association Study Identifies Five New Breast Cancer Susceptibility Loci. Nature Genetics, 42, 504-507. https://doi.org/10.1038/ng.586

[47] Gayther, S.A., et al. (2007) Tagging Single Nucleotide Polymorphisms in Cell Cycle 
Control Genes and Susceptibility to Invasive Epithelial Ovarian Cancer. Cancer Research, 67, 3027-3035. https://doi.org/10.1158/0008-5472.CAN-06-3261

[48] Yeh, I. and Bastian, B.C. (2009) Genome-Wide Associations Studies for Melanoma and Nevi. Pigment Cell \& Melanoma Research, 22, 527.

https://doi.org/10.1111/j.1755-148X.2009.00622.x

[49] Cugino, D., et al. (2012) Type 2 Diabetes and Polymorphisms on Chromosome 9p21: A Meta-Analysis. Nutrition, Metabolism and Cardiovascular Diseases, 22, 619-625. https://doi.org/10.1016/j.numecd.2010.11.010

[50] Emanuele, E., et al. (2011) Chromosome 9p21.3 Genotype Is Associated with Vascular Dementia and Alzheimer's Disease. Neurobiology of Aging, 32, 1231-1235. https://doi.org/10.1016/j.neurobiolaging.2009.07.003

[51] Congrains, A., et al. (2013) ANRIL: Molecular Mechanisms and Implications in Human Health. International Journal of Molecular Sciences, 14, 1278-1292. https://doi.org/10.3390/ijms14011278

[52] Wan, G., et al. (2013) Long Non-Coding RNA ANRIL (CDKN2B-AS) Is Induced by the ATM-E2F1 Signaling Pathway. Cellular Signalling, 25, 1086-1095. https://doi.org/10.1016/j.cellsig.2013.02.006

[53] Kotake, Y., et al. (2011) Long Non-Coding RNA ANRIL Is Required for the PRC2 Recruitment to and Silencing of $\mathrm{p} 15^{\mathrm{INK} 4 \mathrm{~B}}$ Tumor Suppressor Gene. Oncogene, 30, 1956-1962. https://doi.org/10.1038/onc.2010.568

[54] Qiu, J.-J., et al. (2016) The Long Non-Coding RNA ANRIL Promotes Proliferation and Cell Cycle Progression and Inhibits Apoptosis and Senescence in Epithelial Ovarian Cancer. Oncotarget, 7, 32478. https://doi.org/10.18632/oncotarget.8744

[55] Naemura, M., et al. (2015) Long Noncoding RNA ANRIL Regulates Proliferation of Non-Small Cell Lung Cancer and Cervical Cancer Cells. Anticancer Research, 35, 5377-5382.

[56] Schuster-Gossler, K., et al. (1998) The Mouse Gtl2 Gene Is Differentially Expressed during Embryonic Development, Encodes Multiple Alternatively Spliced Transcripts, and May Act as an RNA. Developmental Dynamics, 212, 214-228. https://doi.org/10.1002/(SICI)1097-0177(199806)212:2<214::AID-AJA6>3.0.CO;2-K

[57] Zhang, X., et al. (2003) A Pituitary-Derived MEG3 Isoform Functions as a Growth Suppressor in Tumor Cells. The Journal of Clinical Endocrinology \& Metabolism, 88, 5119-5126. https://doi.org/10.1210/jc.2003-030222

[58] Zhao, J., et al. (2005) Hypermethylation of the Promoter Region Is Associated with the Loss of MEG3 Gene Expression in Human Pituitary Tumors. The Journal of Clinical Endocrinology \& Metabolism, 90, 2179-2186. https://doi.org/10.1210/jc.2004-1848

[59] Benetatos, L., et al. (2008) Promoter Hypermethylation of the MEG3 (DLK1/MEG3) Imprinted Gene in Multiple Myeloma. Clinical Lymphoma and Myeloma, 8, 171175. https://doi.org/10.3816/CLM.2008.n.021

[60] Vousden, K.H. and Prives, C. (2009) Blinded by the Light: The Growing Complexity of p53. Cell, 137, 413-431. https://doi.org/10.1016/j.cell.2009.04.037

[61] Benetatos, L., Vartholomatos, G. and Hatzimichael, E. (2011) MEG3 Imprinted Gene Contribution in Tumorigenesis. International Journal of Cancer, 129, 773 779. https://doi.org/10.1002/ijc.26052

[62] Kruer, T.L., et al. (2016) Expression of the lncRNA Maternally Expressed Gene 3 (MEG3) Contributes to the Control of Lung Cancer Cell Proliferation by the $\mathrm{Rb}$ Pathway. PLoS ONE, 11, e0166363. https://doi.org/10.1371/journal.pone.0166363 
[63] Qin, R., et al. (2013) Long Non-Coding RNA MEG3 Inhibits the Proliferation of Cervical Carcinoma Cells through the Induction of Cell Cycle Arrest and Apoptosis. Neoplasma, 60, 486-492. https://doi.org/10.4149/neo_2013_063

[64] Zhang, J., et al. (2016) Long Noncoding RNA MEG3 Is Downregulated in Cervical Cancer and Affects Cell Proliferation and Apoptosis by Regulating miR-21. Cancer Biology \& Therapy, 17, 104-113. https://doi.org/10.1080/15384047.2015.1108496

[65] Schneider, C., King, R.M. and Philipson, L. (1988) Genes Specifically Expressed at Growth Arrest of Mammalian Cells. Cell, 54, 787-793. https://doi.org/10.1016/S0092-8674(88)91065-3

[66] Pickard, M.R. and Williams, G.T. (2016) The Hormone Response Element Mimic Sequence of GAS5 lncRNA Is Sufficient to Induce Apoptosis in Breast Cancer Cells. Oncotarget, 7, 10104. https://doi.org/10.18632/oncotarget.7173

[67] Shi, X., et al. (2015) A Critical Role for the Long Non-Coding RNA GAS5 in Proliferation and Apoptosis in Non-Small-Cell Lung Cancer. Molecular Carcinogenesis, 54, E1-E12. https://doi.org/10.1002/mc.22120

[68] Pickard, M., Mourtada-Maarabouni, M. and Williams, G. (2013) Long Non-Coding RNA GAS5 Regulates Apoptosis in Prostate Cancer Cell Lines. Biochimica et Biophysica Acta (BBA) - Molecular Basis of Disease, 1832, 1613-1623. https://doi.org/10.1016/j.bbadis.2013.05.005

[69] Liu, Z., et al. (2013) Downregulation of GAS5 Promotes Bladder Cancer Cell Proliferation, Partly by Regulating CDK6. PLOS ONE, 8, e73991.

https://doi.org/10.1371/journal.pone.0073991

[70] Fraser, M., Bai, T. and Tsang, B.K. (2008) Akt Promotes Cisplatin Resistance in Human Ovarian Cancer Cells through Inhibition of p53 Phosphorylation and Nuclear Function. International Journal of Cancer, 122, 534-546. https://doi.org/10.1002/ijc.23086

[71] Wen, Q., et al. (2017) Long Noncoding RNA GAS5, Which Acts as a Tumor Suppressor via MicroRNA 21, Regulates Cisplatin Resistance Expression in Cervical Cancer. International Journal of Gynecological Cancer, 27, 1096. https://doi.org/10.1097/IGC.0000000000001028

[72] Qiu, M., et al. (2014) CCAT2 Is a Lung Adenocarcinoma-Specific Long Non-Coding RNA and Promotes Invasion of Non-Small Cell Lung Cancer. Tumor Biology, 35, 5375-5380. https://doi.org/10.1007/s13277-014-1700-Z

[73] Redis, R.S., et al. (2013) CCAT2, a Novel Long Non-Coding RNA in Breast Cancer: Expression Study and Clinical Correlations. Oncotarget, 4, 1748. https://doi.org/10.18632/oncotarget.1292

[74] Wang, C.-Y., et al. (2015) Long Non-Coding RNA CCAT2 Is Up-Regulated in Gastric Cancer and Associated with Poor Prognosis. International Journal of Clinical and Experimental Pathology, 8, 779.

[75] Huang, S., et al. (2016) The Long Non-Coding RNA CCAT2 Is Up-Regulated in Ovarian Cancer and Associated with Poor Prognosis. Diagnostic Pathology, 11, 49. https://doi.org/10.1186/s13000-016-0499-x

[76] Nan, H., et al. (2013) Aspirin Use, 8q24 Single Nucleotide Polymorphism rs6983 267, and Colorectal Cancer According to CTNNB1 Alterations. Journal of the National Cancer Institute, 105, 1852-1861. https://doi.org/10.1093/jnci/djt331

[77] Peng, Y., et al. (2017) The Crosstalk between MicroRNAs and the Wnt/ $\beta$-Catenin Signaling Pathway in Cancer. Oncotarget, 8, 14089. https://doi.org/10.18632/oncotarget.12923

[78] Rennoll, S. and Yochum, G. (2015) Regulation of Myc Gene Expression by Aberrant 
Wnt/ $\beta$-Catenin Signaling in Colorectal Cancer. World Journal of Biological Chemistry, 6, 290. https://doi.org/10.4331/wjbc.v6.i4.290

[79] Cai, Y., He, J. and Zhang, D. (2015) Long Noncoding RNA CCAT2 Promotes Breast Tumor Growth by Regulating the Wnt Signaling Pathway. OncoTargets and Therapy, 8, 2657.

[80] Kokka, F., et al. (2013) Surgical Treatment of Stage IA2 Cervical Cancer. The Cochrane Database of Systematic Reviews, 5, CD010870.

[81] Le Wu, L.J., Zhang, W. and Zhang, L. (2016) Roles of Long Non-Coding RNA CCAT2 in Cervical Cancer Cell Growth and Apoptosis. Medical Science Monitor. International Medical Journal of Experimental and Clinical Research, 22, 875. https://doi.org/10.12659/MSM.897754

[82] Xue, M., Chen, W. and Li, X. (2016) Urothelial Cancer Associated 1: A Long Noncoding RNA with a Crucial Role in Cancer. Journal of Cancer Research and Clinical Oncology, 142, 1407-1419. https://doi.org/10.1007/s00432-015-2042-y

[83] Tsang, W.P., et al. (2007) Induction of Drug Resistance and Transformation in Human Cancer Cells by the Noncoding RNA CUDR. RNA, 13, 890-898. https://doi.org/10.1261/rna.359007

[84] Wang, X.-S., et al. (2006) Rapid Identification of UCA1 as a Very Sensitive and Specific Unique Marker for Human Bladder Carcinoma. Clinical Cancer Research, 12, 4851-4858. https://doi.org/10.1158/1078-0432.CCR-06-0134

[85] Wang, F., et al. (2008) UCA1, a Non-Protein-Coding RNA Up-Regulated in Bladder Carcinoma and Embryo, Influencing Cell Growth and Promoting Invasion. FEBS Letters, 582, 1919-1927. https://doi.org/10.1016/j.febslet.2008.05.012

[86] Wang, F., et al. (2015) Upregulated lncRNA-UCA1 Contributes to Progression of Hepatocellular Carcinoma through Inhibition of MiR-216b and Activation of FGFR1/ERK Signaling Pathway. Oncotarget, 6, 7899. https://doi.org/10.18632/oncotarget.3219

[87] Huang, J., et al. (2015) Long Non-Coding RNA UCA1 Promotes Breast Tumor Growth by Suppression of p27 (Kip1). Cell Death \& Disease, 5, e1008. https://doi.org/10.1038/cddis.2013.541

[88] Zheng, Q., et al. (2015) Aberrant Expression of UCA1 in Gastric Cancer and Its Clinical Significance. Clinical and Translational Oncology, 17, 640-646. https://doi.org/10.1007/s12094-015-1290-2

[89] Li, J.-Y., Ma, X. and Zhang, C.-B. (2014) Overexpression of Long Non-Coding RNA UCA1 Predicts a Poor Prognosis in Patients with Esophageal Squamous Cell Carcinoma. International Journal of Clinical and Experimental Pathology, 7, 7938.

[90] Fang, Z., et al. (2014) Increased Expression of the Long Non-Coding RNA UCA1 in Tongue Squamous Cell Carcinomas: A Possible Correlation with Cancer Metastasis. Oral Surgery, Oral Medicine, Oral Pathology and Oral Radiology, 117, 89-95. https://doi.org/10.1016/j.0ooo.2013.09.007

[91] Wang, B., et al. (2017) Expression of Long Noncoding RNA Urothelial Cancer Associated 1 Promotes Cisplatin Resistance in Cervical Cancer. Cancer Biotherapy and Radiopharmaceuticals, 32, 101-110. https://doi.org/10.1089/cbr.2016.2156

[92] Beck-Engeser, G.B., et al. (2008) PVT1-Encoded MicroRNAs in Oncogenesis. Retrovirology, 5, 4. https://doi.org/10.1186/1742-4690-5-4

[93] Guan, Y., et al. (2007) Amplification of PVT1 Contributes to the Pathophysiology of Ovarian and Breast Cancer. Clinical Cancer Research, 13, 5745-5755. https://doi.org/10.1158/1078-0432.CCR-06-2882

[94] Webb, E., Adams, J.M. and Cory, S. (1984) Variant (6:15) Translocation in a Mu- 
rine Plasmacytoma Occurs near an Immunoglobulin $\kappa$ Gene but Far from the Myc Oncogene. Nature, 312, 777-779. https://doi.org/10.1038/312777a0

[95] Graham, M. and Adams, J.M. (1986) Chromosome 8 Breakpoint Far 3' of the CMyc Oncogene in a Burkitt's lymphoma 2:8 Variant Translocation Is Equivalent to the Murine PVT-1 Locus. The EMBO Journal, 5, 2845.

[96] Shtivelman, E. and Bishop, J. (1990) Effects of Translocations on Transcription from PVT. Molecular and Cellular Biology, 10, 1835-1839.

https://doi.org/10.1128/MCB.10.4.1835

[97] Ding, J., et al. (2014) Expression and Clinical Significance of the Long Non-Coding RNA PVT1 in Human Gastric Cancer. OncoTargets and Therapy, 7, 1625. https://doi.org/10.2147/OTT.S68854

[98] Ding, C., et al. (2015) Long Non-Coding RNA PVT1 Is Associated with Tumor Progression and Predicts Recurrence in Hepatocellular Carcinoma Patients. Oncology Letters, 9, 955-963. https://doi.org/10.3892/ol.2014.2730

[99] Huang, C., et al. (2015) Increased Expression of The lncRNA PVT1 Is Associated with Poor Prognosis in Pancreatic Cancer Patients. Minerva Medica, 106, 143-149.

[100] Haverty, P.M., et al. (2009) High-Resolution Analysis of Copy Number Alterations and Associated Expression Changes in Ovarian Tumors. BMC Medical Genomics, 2, 21. https://doi.org/10.1186/1755-8794-2-21

[101] Wang, F., et al. (2014) Oncofetal Long Noncoding RNA PVT1 Promotes Proliferation and Stem Cell-Like Property of Hepatocellular Carcinoma Cells by Stabilizing NOP2. Hepatology, 60, 1278-1290. https://doi.org/10.1002/hep.27239

[102] Takahashi, Y., et al. (2014) Amplification of PVT-1 Is Involved in Poor Prognosis via Apoptosis Inhibition in Colorectal Cancers. British Journal of Cancer, 110, 164171. https://doi.org/10.1038/bjc.2013.698

[103] Cerami, E., et al. (2012) The cBio Cancer Genomics Portal: An Open Platform for Exploring Multidimensional Cancer Genomics Data. Cancer Discovery, 2, 401-404.

[104] Gao, J., et al. (2013) Integrative Analysis of Complex Cancer Genomics and Clinical Profiles Using the cBioPortal. Science Signaling, 6, pl1. https://doi.org/10.1126/scisignal.2004088

[105] Zhang, S., Zhang, G. and Liu, J. (2016) Long Noncoding RNA PVT1 Promotes Cervical Cancer Progression through Epigenetically Silencing miR-200b. Journal of Pathology, Microbiology and Immunology (APMIS), 124, 649-658. https://doi.org/10.1111/apm.12555

[106] Iden, M., et al. (2016) The lncRNA PVT1 Contributes to the Cervical Cancer Phenotype and Associates with Poor Patient Prognosis. PLOS ONE, 11, e0156274. https://doi.org/10.1371/journal.pone.0156274

[107] Liu, Y., et al. (2012) A Genetic Variant in Long Non-Coding RNA HULC Contributes to Risk of HBV-Related Hepatocellular Carcinoma in a Chinese Population. PLoS ONE, 7, e35145. https://doi.org/10.1371/journal.pone.0035145

[108] Yang, X.-J., et al. (2016) Long Noncoding RNA HULC Promotes Colorectal Carcinoma Progression through Epigenetically Repressing NKD2 Expression. Gene, 592, 172-178. https://doi.org/10.1016/j.gene.2016.08.002

[109] Uzan, V.R.M., et al. (2016) High Expression of HULC Is Associated with Poor Prognosis in Osteosarcoma Patients. PLoS ONE, 11, e0156774. https://doi.org/10.1371/journal.pone.0156774

[110] Sun, W., et al. (2016) Roles of Long Noncoding RNAs in Gastric Cancer and Their Clinical Applications. Journal of Cancer Research and Clinical Oncology, 142, 2231-2237. https://doi.org/10.1007/s00432-016-2183-7 